\title{
A stress interpretation scheme applied to lunar gravity and topography data
}

\author{
F. Chambat ${ }^{1}$ and B. Valette ${ }^{2}$ \\ Received 7 May 2007; revised 30 September 2007; accepted 20 November 2007; published 23 February 2008.
}

[1] We present an approach of the inverse gravimetric problem that allows the gravity to be directly related to the deviatoric stresses without any rheological assumptions. In this approach a new set of parameters is considered: (1) the density variations over equipotential surfaces and the height of interfaces above the corresponding equipotential surfaces and (2) the stress difference. The method is applied to lunar topographic and gravimetric data that are interpreted in term of transversally isotropic deviatoric stress within the Moon. It also provides inference on density and crustal thickness variations. The estimated lateral variation in deviatoric stress is about 500 bars within the crust and upper mantle. In the crust, because of topography, the strongest stress differences take place on the far side, with large lateral compressions beneath the south pole-Aitken basin. Vertical compression under the mascons of the nearside is the main feature within the upper mantle.

Citation: Chambat, F., and B. Valette (2008), A stress interpretation scheme applied to lunar gravity and topography data, J. Geophys. Res., 113, E02009, doi:10.1029/2007JE002936.

\section{Introduction}

[2] In this article we apply to the Moon a new interpretation method of topographic and gravimetric data that gives inference on the state of stress within the planet as well as on its density and crustal thickness. This method, which was introduced by Valette [1987], Chambat [1996], and Valette and Chambat [2004], is based upon a reparameterization of the inverse gravimetric problem in terms of stress difference. The approach differs from that of Backus [1967] in the fact that we use Lagrangian perturbations of stress, which simplifies boundary conditions and allows the stress field to be written in its local eigendirections basis. The method relies on the global minimization of the stress difference, which corresponds to a mechanical criterion. We determine the minimum deviatoric stresses compatible with the observed topography and gravity. Minimizing the deviatoric stresses was also considered by Dahlen [1981, 1982] as a possible interpretation of isostasy. In a regional framework, Flesch et al. [2001] showed how to obtain, through a finite element approach, the minimum vertically averaged deviatoric stress field that accounts for the equilibrium equation and a given density model.

[3] The first reason for applying this method to the Moon is that its mass and mean inertia are well known, and that their ratio is close to the one of a homogeneous sphere. It yields relatively well constrained radial density models. The second reason lies in the existence of detailed models of the

\footnotetext{
${ }^{1}$ Laboratoire de Sciences de la Terre, CNRS UMR5570, École Normale Supérieure de Lyon, Université de Lyon, Université Claude Bernard Lyon 1, Lyon, France.

${ }^{2}$ Laboratoire de Géophysique Interne et Tectonophysique, IRD: R157, CNRS, Université de Savoie, Le Bourget-du-Lac, France.

Copyright 2008 by the American Geophysical Union. 0148-0227/08/2007JE002936
}

Moon topography and gravity fields. They were computed, up to spherical harmonics order and degree 70, by Zuber et al. [1994], Lemoine et al. [1997], and Smith et al. [1997] from data of the Clementine lunar orbiting mission and previous missions, and by Konopliv et al. [1998] from the Lunar Prospector spacecraft data.

[4] Several inversion schemes have been applied to lunar data (see Wieczorek [2007] for a review). Zuber et al. [1994] derived crustal thickness variations to first order, assuming that lunar gravity is only due to surface and crustal topographies. Neumann et al. [1996] took the dense mare fill into account and used a more accurate method to evaluate the effect of topography. Wieczorek and Phillips [1997, 1998] and Konopliv et al. [1998] used a spectral analysis to investigate the state of compensation and the structure of the lunar crust. Hikida and Wieczorek [2007] considered polyhedral shape models to invert in the spatial domain for lateral variations of crustal thickness.

[5] More specifically, several interpretations of the positive gravity anomalies linked to large basins, or mascons, have been proposed: variations in crustal topography [e.g., Zuber et al., 1994] variations in intracrustal topography [e.g., Wieczorek and Phillips, 1997, 1998], variations in mare basalt thickness [e.g., Kiefer, 1997], and possibly combinations of such variations [e.g., Neumann et al., 1996].

[6] The method that we introduce here allows for all sources of anomalies, volumetric as well as topographic. The methodology is outlined in section 2. Section 3 is devoted to radial models of density. Sections 4 and 5 present the principles and the results of the inversion.

\section{Gravity as a Function of Stress}

[7] The purpose of this section is first to explain concisely how gravity can be written in term of density variations over equipotential surfaces and why this is more 
pertinent than using the usual variations over spherical surfaces. Then, we will show how to express the gravity as a function of the stress field [see also Valette and Chambat, 2004] without any rheological law. The method consists in solving Poisson's equation:

$$
\Delta \varphi=4 \pi G \rho-2 \Omega^{2},
$$

together with the equilibrium equation:

$$
\operatorname{div} \mathbf{T}=\rho \operatorname{grad} \varphi,
$$

where $\Delta$ is the Laplace operator, $\varphi$ is the gravity potential, $G$ is the gravitational constant, $\rho$ is the density, $\Omega$ is the rotational circular frequency of the planet, and $\mathbf{T}$ is the Cauchy stress tensor. The gravity field is defined by $\mathbf{g}=$ $-\operatorname{grad} \varphi$. Let $[p]$ denote the jump of a parameter $p$ across a closed boundary $\Sigma$ oriented by the unit normal vector field $\mathbf{n}_{\Sigma}$. The boundary conditions are

$$
[\varphi]=0, \quad\left[\mathbf{g} \cdot \mathbf{n}_{\Sigma}\right]=0
$$

and

$$
\left[\mathbf{T}\left(\mathbf{n}_{\Sigma}\right)\right]=0 .
$$

[8] To solve this problem, we use a perturbation method around a spherical reference configuration because (1) equation (2) depends nonlinearly on $\rho$; (2) the shape is involved in the solutions of the equations; and (3) the planets have a quasi-spherical symmetry.

[9] As usual in a Lagrangian description [e.g., Chambat and Valette, 2001], each point $\mathbf{x}$ in the real configuration is linked to a point $\mathbf{a}$ in the reference configuration by a bijective mapping $\mathbf{a} \rightarrow \mathbf{x}(\mathbf{a})$ (see Figure 1). We can choose this mapping in such a way that (1) the difference in position corresponds to a radial vector $\mathbf{x}-\mathbf{a}=\xi \mathbf{e}_{r}$ where $\mathbf{e}_{r}$ is the radial unit vector and $\xi$ a scalar; and (2) the interfaces of the reference configuration are mapped onto that of the real one. Thus the Lagrangian displacement $\xi$ is uniquely determined on the boundary and the interfaces.

[10] We may use either Eulerian or Lagrangian mathematical perturbations. Indeed, each parameter $p$ in the real configuration can be expressed as

$$
p(\mathbf{a})=p_{0}+\delta_{e} p,
$$

when $p$ is considered at a point $\mathbf{a}$, or as

$$
p(\mathbf{x})=p_{0}+\delta_{l} p,
$$

when $p$ is considered at the 'displaced' point $\mathbf{x}=\mathbf{a}+\xi \mathbf{e}_{r}$ of the body. $p_{0}$ is the value in the reference configuration at point a, $\delta_{e} p$ its Eulerian perturbation and $\delta_{l} p$ its Lagrangian perturbation. Because the perturbation equations are written with respect to the reference configuration, there is no possible confusion between the notations $p$ and $p_{0}$ so that, from now on, the subscript 0 will be dropped. The displacement is assumed to be small and radial, thus the perturbations, correct to first order in $\xi$, are linked by

$$
\delta_{l} p=\delta_{e} p+\left(\partial_{r} p\right) \xi
$$

where $\partial_{r}$ denotes the derivation with respect to radius $r$. We can perform perturbations of equations (1) and (2) and expand the perturbed quantities into real spherical harmonics, normalized as by Chambat and Valette [2001].

\subsection{Density and Poisson's Equation}

[11] The classical way to solve Poisson's equation in a quasi-spherical geometry is to expand its Eulerian firstorder perturbation into spherical harmonics. At each radius $r$ and for each degree $\ell$ and order $m$, this yields

$$
\left(\partial_{r}^{2}+\frac{2}{r} \partial_{r}-\frac{\ell(\ell+1)}{r^{2}}\right) \delta_{e} \varphi_{\ell}^{m}(r)=4 \pi G \delta_{e} \rho_{\ell}^{m}(r)-2 \Omega^{2} \delta_{\ell}^{0},
$$

where $\delta_{e} \varphi_{\ell}^{m}$ and $\delta_{e} \rho_{\ell}^{m}$ are the degree $\ell$, order $m$ coefficients of the expansion of the Eulerian perturbations of the potential and density, respectively, and $\delta_{\ell}^{0}$ denotes the Kronecker symbol. From now on, the subscript $\ell$, the superscript $m$, the dependence in $r$, and the case $\ell=0$ will be omitted. The associated boundary conditions are

$$
\left[\delta_{e} \varphi\right]=0, \quad\left[\partial_{r} \delta_{e} \varphi+4 \pi G \rho \xi_{\Sigma}\right]=0,
$$

where $\xi_{\Sigma}$ is the height of the interface above the corresponding sphere of mean radius $r_{\Sigma}$. Because of the harmonicity of the gravitational potential outside the Moon, the boundary condition at the surface of $r=b$ may be written as

$$
\partial_{r} \delta_{e} \varphi+(\ell+1) \delta_{e} \varphi / b+4 \pi G \rho \xi_{\Sigma}=\frac{5 \Omega^{2} b}{3} \delta_{\ell}^{2} \delta_{m}^{0},
$$

where $\delta_{\ell}^{2}$ and $\delta_{m}^{0}$ denote Kronecker symbols. The wellknown solution of equation (8), with boundary conditions (9) and (10), may be expressed at $r=b$ as [e.g., Kaula, 1968]

$$
\begin{aligned}
\delta_{e} \varphi(b)= & \frac{4 \pi G}{(2 \ell+1) b^{\ell+1}}\left(\int_{0}^{b} \delta_{e} \rho(r) r^{\ell+2} \mathrm{~d} r\right. \\
& \left.-\sum_{r=r_{\Sigma}}[\rho] \xi_{\Sigma} r^{\ell+2}\right)+\frac{\Omega^{2} b^{2}}{3 \sqrt{5}} \delta_{\ell}^{2} \delta_{m}^{0} .
\end{aligned}
$$

The three terms of this relation account for the volumetric, surficial, and centrifugal potential contributions, respectively.

[12] The drawback of this parameterization is that the hydrostatic term does not clearly appear. This leads, for instance, to some difficulties in expressing the hydrostaticity of the fluid core [e.g., Piersanti et al., 2001]. We propose a new parameterization which is more significant for the gravimetric problem, and relies on the three following variables:

$$
\begin{gathered}
h_{\varphi}=\frac{\delta_{e} \varphi}{g}, \quad h_{\Sigma}=\xi_{\Sigma}-h_{\varphi}, \\
\delta_{\varphi} \rho=\delta_{e} \rho+\left(\partial_{r} \rho\right) h_{\varphi},
\end{gathered}
$$

where $g=\mathbf{g} \cdot \mathbf{e}_{r}$ stands for the (negative) radial gravity in the reference configuration. Correct to first order, $h_{\varphi}$ represents the equipotential height above the reference sphere of radius 


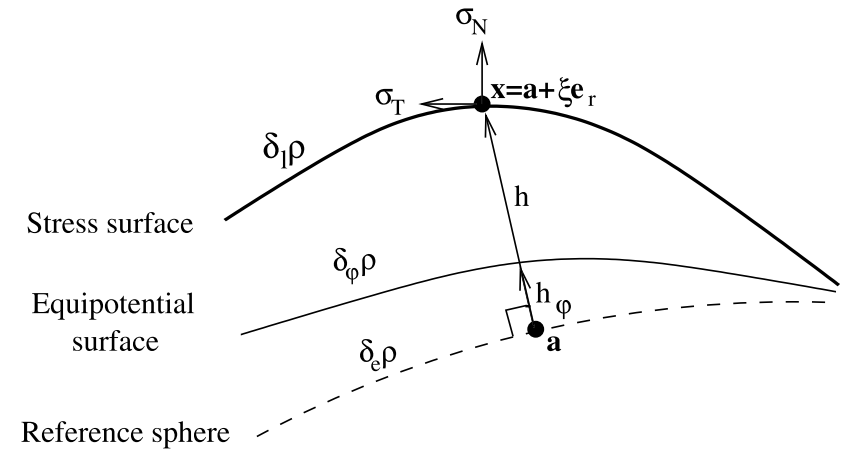

Figure 1. Different surfaces and parameters used in this study: the reference sphere of radius $r$, the equipotential surface corresponding to the sphere, and the stress surface, i.e., the surface orthogonal to the quasi-radial eigendirection of the stress. The height of the equipotential surface with respect to the reference sphere is denoted by $h_{\varphi}$, and the height difference between the stress surface and the equipotential surface, i.e., the altitude, is denoted by $h$. The lateral variations in density may be considered over the reference sphere $\left(\delta_{e} \rho\right)$, over the equipotential surface $\left(\delta_{\varphi} \rho\right)$, or over the stress surface $\left(\delta_{l} \rho\right)$.

$r$, and corresponds at the external surface to the geoidal height. $h_{\Sigma}$ is the height of an interface above the corresponding equipotential surface and coincides with the usual altitude at the external surface. $\delta_{\varphi} \rho$ represents the lateral variation in density over equipotential surfaces (see Figure 1).

[13] One easily shows that with these variables equation (8) takes the form

$$
\left\{\partial_{r}^{2}-\frac{2}{r}(1-3 \gamma) \partial_{r}-\frac{k^{2}}{r^{2}}\right\} h_{\varphi}=\frac{4 \pi G}{g} \delta_{\varphi} \rho,
$$

while the boundary conditions become

$$
\left[h_{\varphi}\right]=0 \quad, \quad\left[r \partial_{r} h_{\varphi}\right]=3[\gamma] h_{\Sigma}
$$

at $r=r_{\Sigma}$, and

$$
\left(b \partial_{r} h_{\varphi}+(\ell-1) h_{\varphi}\right)=3 \gamma h_{\Sigma}+\frac{\sqrt{5}}{3} \frac{\Omega^{2} b^{2}}{g(b)} \delta_{\ell}^{2} \delta_{m}^{0}
$$

at $r=b$, and where $k=\sqrt{(\ell-1)(\ell+2)}$ is a horizontal wave number, while

$$
\gamma=-\frac{4 \pi G \rho r}{3 g}=\frac{\rho}{\rho_{2}}
$$

is the ratio of the reference density $\rho$ at radius $r$ to the mean density $\rho_{2}(r)=3 \int_{0}^{r} \rho(s) s^{2} \mathrm{~d} s / r^{3}$ inside the sphere of radius $r$.

[14] Equation (14) may be rewritten as a first-order system involving $h_{\varphi}(r)$ without derivative of $\rho$ :

$$
\begin{aligned}
\partial_{r}\left(\begin{array}{c}
h_{\varphi} \\
r \partial_{r} h_{\varphi}
\end{array}\right)= & \frac{1}{r}\left(\begin{array}{cc}
0 & 1 \\
k^{2} & 3(1-2 \gamma)
\end{array}\right)\left(\begin{array}{c}
h_{\varphi} \\
r \partial_{r} h_{\varphi}
\end{array}\right) \\
& -3 \gamma\left(\frac{\delta_{\varphi}^{0} \rho}{\rho}\right) .
\end{aligned}
$$

[15] It is then straightforward to show that the solution at $r=b$ is

$$
\begin{aligned}
h_{\varphi}(b)= & -\frac{4 \pi G}{g^{2}(b) b}\left(\int_{0}^{b} x g r^{2} \delta_{\varphi} \rho \mathrm{d} r-\sum_{r=r_{\Sigma}} x g r_{\Sigma}^{2}[\rho] h_{\Sigma}\right) \\
& +\frac{\sqrt{5}}{3} \frac{\Omega^{2} b^{2}}{g(b)} \delta_{\ell}^{2} \delta_{m}^{0} x(b),
\end{aligned}
$$

where $x$ is a dimensionless function of the radius that must be numerically estimated using a reference density model. More precisely, $\left(x, r \partial_{r} x\right)(r)$ is the continuous solution of the homogeneous system corresponding to (18), i.e., with $\delta_{\varphi} \rho \equiv 0$ in (18) and $h_{\Sigma} \equiv 0$ in (15), with the following conditions: for $\ell>1$

$$
\left(\begin{array}{c}
x \\
r \partial_{r} x
\end{array}\right) \underset{r \sim 0}{\sim} C\left(\begin{array}{c}
1 \\
l-1
\end{array}\right) r^{\ell-1}
$$

at the center ( $C$ denotes a constant), and

$$
\left(r \partial_{r} x+(\ell-1) x\right)(b)=1
$$

at the external boundary, while for $\ell=1, x(r) \equiv 1$. It can be shown that $x(r)$ remains close to $r^{\ell-1}$ [Chambat and Valette, 2001; Valette and Chambat, 2004].

\subsection{Hydrostatic Equilibrium}

[16] In the hydrostatic case, equation (2) takes the form

$$
\operatorname{grad} p=-\rho \operatorname{grad} \varphi,
$$

where $p$ denotes the pressure. This implies that equipotential and equipressure surfaces coincide with each other and are equidensity surfaces. It also implies that the interfaces are equiparameter surfaces. This means that $\delta_{\varphi} \rho$ vanishes in hydrostatic regions and that $h_{\Sigma}$ vanishes when each side of the boundary $\Sigma$ is hydrostatic. Thus, for each $(\ell, m)$, relation (19) provides a new expression of the gravity field as a function of perturbations that are representative of the state of deviatoric stresses. If the whole planet is hydrostatic, the only term that remains is the last one, which contains $\Omega^{2}$. This term corresponds to the classical hydrostatic ellipticity term of degree $(\ell, m)=(2,0)$. It shows that the homogeneous system corresponding to (18) is an alternative way of writing Clairaut's differential equation. It also shows that in order to model the lateral variations of potential and topography, the deviatoric stresses must be taken into account. For this purpose we now show that $\delta_{\varphi} \rho$ and $h_{\Sigma}$ can be expressed in term of stress difference.

\subsection{Taking Stress Differences Into Account}

[17] It is possible to write the potential as a function of the state of stress without any hypothesis [Chambat, 1996; Valette and Chambat, 2004], but since the stress tensor involves six independent parameters, this yields a very underdetermined inverse problem. We propose here to make a simplification at first. At an interface with an hydrostatic region, like the external surface, the normal direction to the surface is an eigendirection of the stress tensor, due to the boundary condition (4). Moreover in the spherical reference 
configuration the tensor is transversally isotropic with respect to the radial direction. Thus we will assume that the stress tensor remains transversally isotropic around a quasi-vertical unit vector field $\mathbf{n}$, so that it may be written as

$$
\begin{gathered}
\mathbf{T}=\sigma_{N} \mathbf{n} \otimes \mathbf{n}+\sigma_{T} \mathbf{P}_{T} \\
\mathbf{T}=\frac{1}{3}\left(\sigma_{N}+2 \sigma_{T}\right) \mathbf{I}-\frac{2}{3}\left(\sigma_{T}-\sigma_{N}\right) \mathbf{n} \otimes \mathbf{n}+\frac{1}{3}\left(\sigma_{T}-\sigma_{N}\right) \mathbf{P}_{T}
\end{gathered}
$$

where $\sigma_{N}$ and $\sigma_{T}$ denote the normal and tangential eigenvalues of $\mathbf{T}, \otimes$ the tensor product, I the identity tensor and $\mathbf{P}_{T}$ the projector onto the local plane perpendicular to $\mathbf{n}$. The surface with normal $\mathbf{n}$ is unknown and its height above the equipotential will be noted $h$.

[18] The stress and the density are related by the equilibrium equation (2). To make this relation explicit, we express the Lagrangian perturbation of (2) around the spherical reference. This linearization reads (see Appendix A)

$$
\begin{aligned}
\operatorname{grad} \delta_{l} \sigma_{N} & +\operatorname{grad}_{T} \delta_{l}\left(\sigma_{T}-\sigma_{N}\right)-\frac{2}{r} \mathbf{e}_{r} \delta_{l}\left(\sigma_{T}-\sigma_{N}\right) \\
& +\left(\delta_{\varphi} \rho\right) \mathbf{g}+\operatorname{grad}(\rho g h)=0
\end{aligned}
$$

where $\operatorname{grad}_{T}=\mathbf{P}_{T} \cdot \operatorname{grad}$ is the tangential, or surface, gradient, and $\delta_{l} \sigma_{N}$ and $\delta_{l} \sigma_{T}$ are the lateral variations in normal and tangential stress. For each degree $(\ell \neq 0)$, the tangential and radial components of equation (25) are

$$
\begin{gathered}
\rho g h=-\delta_{l} \sigma_{T} \\
\delta_{\varphi} \rho=\frac{1}{g r^{2}} \partial_{r}\left(\delta_{l}\left(\sigma_{T}-\sigma_{N}\right) r^{2}\right),
\end{gathered}
$$

respectively. The first relation, which can be rewritten as $\delta_{\varphi} \sigma_{T}=0$, shows that the tangential stress is constant over equipotential surfaces. The boundary conditions are (see Appendix B)

$$
[\rho] g h_{\Sigma}=-\left[\delta_{l}\left(\sigma_{T}-\sigma_{N}\right)\right] .
$$

In particular, at the external surface the condition is

$$
\rho g h_{\Sigma}(b)=-\delta_{l}\left(\sigma_{T}-\sigma_{N}\right)(b)
$$

Thus, by equations (27) and (28) the variations in density are linked to the variations in stress difference, and the altitude of interfaces are related to the jump in stress difference.

[19] Upon substituting relations (27) and (28) into equation (19) and integrating by parts we finally obtain the expression of the height of the equipotential surface as a function of the stress difference:

$$
\begin{aligned}
h_{\varphi}(b) & =\frac{4 \pi G}{b g^{2}(b)} \int_{0}^{b} \delta_{l}\left(\sigma_{T}-\sigma_{N}\right) r^{2} \partial_{r} x \mathrm{~d} r \\
& +\frac{\sqrt{5}}{3} \frac{\Omega^{2} b^{2}}{g(b)} \delta_{\ell}^{2} \delta_{m}^{0} x(b) .
\end{aligned}
$$

The relations (29) and (30) allow us to infer the stress difference from $h_{\varphi}(b)$ and $h_{\Sigma}(b)$. It is important to note that this stress difference $\delta_{l}\left(\sigma_{T}-\sigma_{N}\right)$ represents three times the tangential eigenvalue of the deviatoric stress, as equation (24) shows. From $\delta_{l}\left(\sigma_{T}-\sigma_{N}\right)$ we can derive information on density and topography through equations (27) and (28).

[20] Let us also notice that even if the assumption of transverse isotropy is not exactly verified, it is always possible to define the function $\delta_{l}\left(\sigma_{T}-\sigma_{N}\right)$ obeying equations (27) and (28), which leads to the expression (30). Thus $\delta_{l}\left(\sigma_{T}-\sigma_{N}\right)$ may at least be considered as an intermediate variable, which vanishes with the deviatoric stress, and allows for any density distribution and any variation in topography.

[21] An extension of the method would consist in relaxing the hypothesis of tangential isotropy. We have shown [Valette and Chambat, 2004, section 4.4] that in this case the lateral variations in density and potential depend on two scalar fields instead of only one. This may be compared to the method followed by Flesch et al. [2001], one of the main differences being that they consider a completely known density model, whereas our approach provides inference on lateral density variations.

\section{Reference Density Model}

[22] For the purpose of inversion, a reference model $\rho(r)$ is required to compute the function $x(r)$ involved in the kernel of equation (30).

[23] The mean radius is given by the Clementine altimetry [Smith et al., 1997]:

$$
b=1737.10 \pm 0.05 \mathrm{~km} \text {. }
$$

We assign here a $50 \mathrm{~m}$ error in order to match the $1737.14 \mathrm{~km}$ value appearing in the Clementine numeric model file gltm 2 bsh.tab. The mass is derived through the $G M$ value from the Lunar Prospector data [Konopliv et al., 1998] and through that of $G$ from Mohr and Taylor [2005](available at http://physics.nist.gov/cuu/):

$$
\mathcal{M}=(7.3459 \pm 0.0010) \times 10^{22} \mathrm{~kg}
$$

The mean inertia coefficient is derived from Lunar Laser Ranging and Lunar Prospector data. After a renormalization of the value given by Konopliv et al. [1998] done to introduce the physical radius $b$ instead of the conventional radius $R$ we obtain:

$$
\frac{\mathcal{I}}{M b^{2}}=0.3935 \pm 0.0002
$$

Defining the normalized density moments $\rho_{2}$ and $\rho_{4}$ as

$$
\rho_{2}(b)=\frac{3}{b^{3}} \int_{0}^{b} \rho r^{2} \mathrm{~d} r \quad \rho_{4}(b)=\frac{5}{b^{5}} \int_{0}^{b} \rho r^{4} \mathrm{~d} r
$$

the mass and inertia data may be expressed as

$$
\rho_{2}(b)=3 M / 4 \pi b^{3}=3345.7 \pm 0.5 \mathrm{~kg} / \mathrm{m}^{3}
$$

$$
\rho_{4}(b)=15 I / 8 \pi b^{5}=3291.3 \pm 2.4 \mathrm{~kg} / \mathrm{m}^{3} .
$$



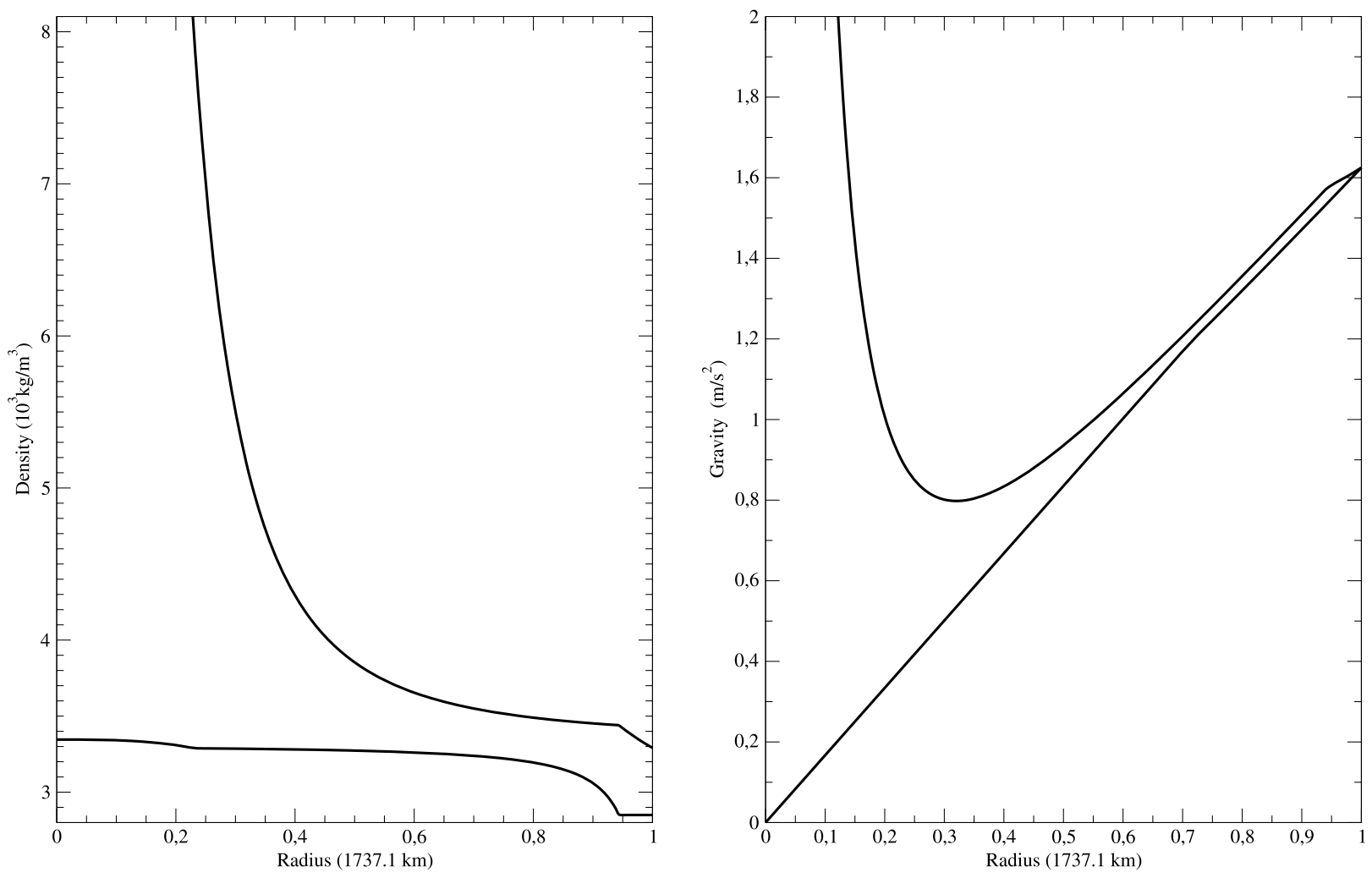

Figure 2. (left) Upper and lower bounds of the density based on mass and inertia. (right) Same as Figure 2 (left) except for gravity [Valette, 2000].

[24] Taking account of the fact that mare basalt of density $3300 \mathrm{~kg} / \mathrm{m}^{3}$ covers about one fifth of the Moon surface with density $2800 \mathrm{~kg} / \mathrm{m}^{3}$ [Solomon and Toksöz, 1973], we choose a mean surface density $\rho(b)=2850 \mathrm{~kg} / \mathrm{m}^{3}$. The existence of a dense core is still debated. Upon the assumption that an iron core exists, as suggested by the remanent magnetism or by the analysis of the lunar rotational dissipation [Williams et al., 2001], the density at the center can be estimated from high-pressure experimental data [Boehler et al., 1990]. Interpolating these data through an equation of state and assuming the temperature and pressure at the center to be in the range 1000-2000 K [Solomon and Toksöz, 1973] and about $6 \mathrm{GPa}$ gives a value for the density of pure solid iron ranging from 7700 to $8100 \mathrm{~kg} / \mathrm{m}^{3}$. We thus choose $\rho(0)=$ $7900 \mathrm{~kg} / \mathrm{m}^{3}$.

[25] Another important source of information is the analysis of the Apollo seismic data by Nakamura [1983], Khan et al. [2000], Khan and Mosegaard [2002], and Lognonné et al. [2003]. Khan et al. [2000] inferred a crustal thickness of $45 \mathrm{~km}$, and a piecewise seismic velocity model in the mantle with values ranging from $V_{P}=8 \mathrm{~km} / \mathrm{s}$ and $V_{S}=4 \mathrm{~km} / \mathrm{s}$ at depth shallower than $560 \mathrm{~km}$ up to $V_{P}=$ $11 \mathrm{~km} / \mathrm{s}$ and $V_{S}=6 \mathrm{~km} / \mathrm{s}$ down to a depth of $1100 \mathrm{~km}$. Because of the localization of the Apollo seismic stations, the mean crustal thickness may be increased to $50 \mathrm{~km}$ to take lateral variations into account.

[26] The global upper and lower bounds (7900 and $2850 \mathrm{~kg} / \mathrm{m}^{3}$, respectively) and the hypothesis that density increases with depth lead to relatively close bounds on density as well as on its moments of order 2 and 4 at each depth [Stieltjes, 1884; Bills and Rubincam, 1995; Valette,
2000]. In Figure 2 the bounding curves corresponding to the values listed above are displayed. They show that the maximum radius of an iron core is about $400 \mathrm{~km}$, that the upper bound of the density jump at the crust-mantle

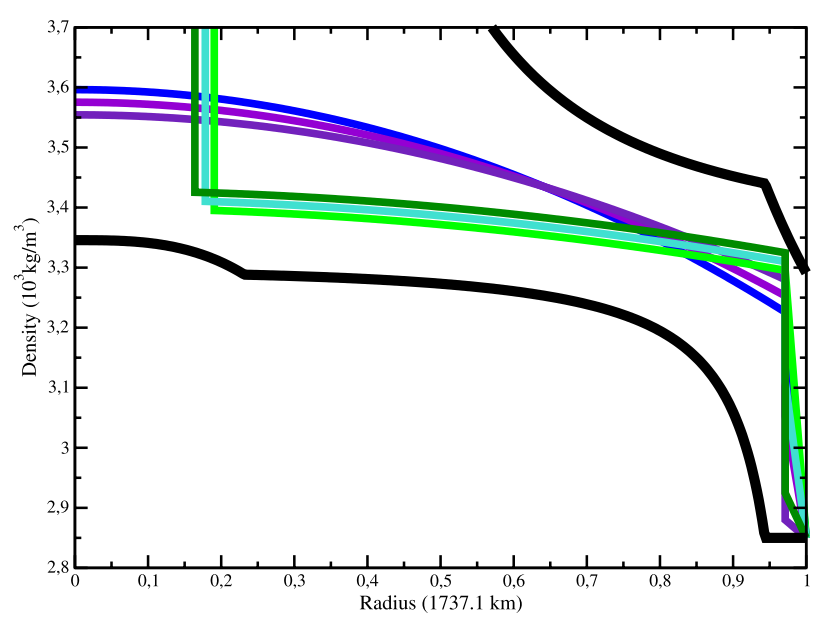

Figure 3. Two classes of density models, one without core (purple lines) and the other with a pure iron core and an adiabatic mantle (green lines). Within each of these two classes, the models differ from each other only by the choice of the density jump at the base of the crust: $\delta=0,200$, or $400 \mathrm{~kg} / \mathrm{m}^{3}$, respectively. One can see that the main influence of this choice on the models is the slope in the crust. The density limits of Figure 2 are also drawn (black lines). 


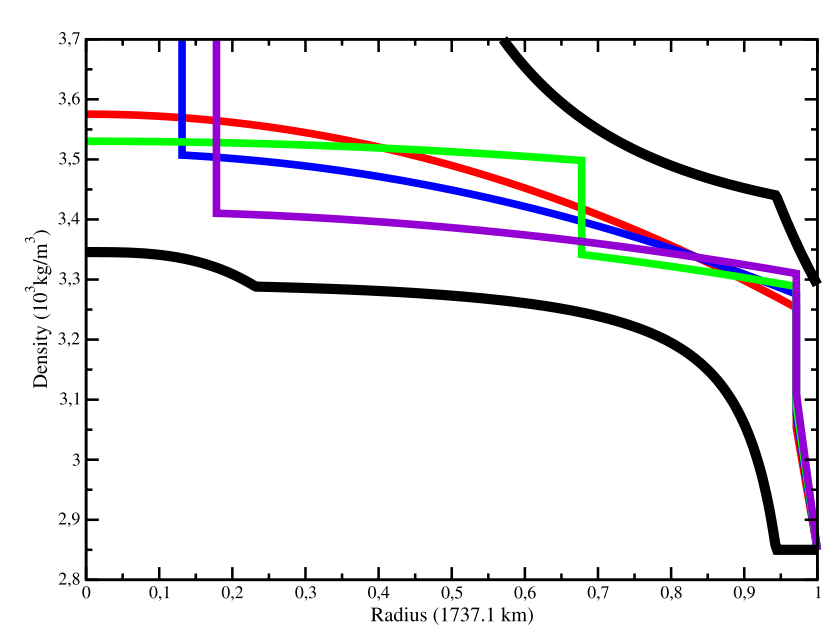

Figure 4. Four density models described in the text and the density limits. All the models have a median jump of $200 \mathrm{~kg} / \mathrm{m}^{3}$ at the base of the crust. One model is approximately adiabatic everywhere but has a $560 \mathrm{~km}$ depth discontinuity (green line). Two models have a dense core $\left(7900 \mathrm{~kg} / \mathrm{m}^{3}\right)$ and correspond to $\beta=110 \mathrm{~kg} / \mathrm{m}^{3}$ (the adiabatic value, purple line) and $250 \mathrm{~kg} / \mathrm{m}^{3}$ (blue line), respectively. The fourth model corresponds to the limit of a vanishing core radius with $\beta=342 \mathrm{~kg} / \mathrm{m}^{3}$ (red line). The black lines correspond to the density limits.

boundary is $\delta=-[\rho] \leq 511 \mathrm{~kg} / \mathrm{m}^{3}$ and that the gravity $g$ can be considered to be a linear function of radius within the upper mantle.

[27] Another possible assumption is that the mantle is adiabatic. It leads to Adams-Williamson relation $\partial_{r} \rho / \rho=g / c^{2}$ with $c^{2}=V_{P}^{2}-4 V_{S}^{2} / 3$. Since $c$ and $g / r$ are almost constant in the mantle, integration around a reference radius $r_{0}$ gives with a very good accuracy, $\rho(r)=\alpha-\beta(r / b)^{2}$ where $\beta=\rho\left(r_{0}\right)|g / r| b^{2} / 2 c^{2}$. For the upper mantle $\beta \simeq$ $110 \mathrm{~kg} / \mathrm{m}^{3}$. For the lower mantle, its value decreases to about $70 \mathrm{~kg} / \mathrm{m}^{3}$ if there is no dense core, i.e., if $\mathrm{g} / \mathrm{r}$ remains approximatively constant down to the center. On the contrary, the value for the lower mantle increases to about $150 \mathrm{~kg} / \mathrm{m}^{3}$ if an iron core does exist.

[28] We have explored different types of models. The simplest one consists of two layers, namely, the mantle and the crust. Within the mantle we have set $\rho(r)=\alpha-\beta(r / b)^{2}$, while within the crust the density is assumed to be a linear function of the radius. The value of the density being imposed at the surface, three parameters, $\alpha, \beta$ and the jump $\delta$ remain to be determined from the two data $\rho_{2}(b)$ and $\rho_{4}(b) . \delta$ can vary between 0 and $435 \mathrm{~kg} / \mathrm{m}^{3}$, while $\beta$ and $\alpha$ decrease from 393 to $282 \mathrm{~kg} / \mathrm{m}^{3}$ and from 3596 to $3551 \mathrm{~kg} / \mathrm{m}^{3}$, respectively. Figure 3 shows that the main difference between the models essentially lies in the slope within the crust according to the $\delta$ value. Since it is the same for all types of models, we have imposed a median value of $200 \mathrm{~kg} / \mathrm{m}^{3}$ for $\delta$. In addition, the results for $\beta$ show that the upper mantle cannot be assumed to be adiabatic, unless one assumes an iron core or a discontinuity in the mantle. We thus consider a second type of model consisting of a linear crust overlying two layers within each of which $\rho(r)=\alpha-\beta(r / b)^{2}$. In the case of a pure iron core $\alpha_{\text {core }}$ is known and, since $\delta$ is fixed, $\beta_{\text {core }}, r_{\text {core }}, \alpha$ and $\beta$ remain to be determined. As the value of $\beta_{\text {core }}$ has practically no effect on the model, we take $\beta_{\text {core }}=260 \mathrm{~kg} / \mathrm{m}^{3}$, which is approximatively the adiabatic value. Making $\beta$ increase from the adiabatic value of $110 \mathrm{~kg} / \mathrm{m}^{3}$ to the limit of $342 \mathrm{~kg} / \mathrm{m}^{3}$, the radius $r_{\text {core }}$ decreases from 310 to $0 \mathrm{~km}$, while $\alpha$ ranges from 3414 to $3574 \mathrm{~kg} / \mathrm{m}^{3}$. Since the adiabatic value can be considered as a lower bound for $\beta$, this shows that the radius of a pure solid iron core cannot practically exceed $310 \mathrm{~km}$. This range of models is fully illustrated by the two models corresponding to $\beta=110$ and $250 \mathrm{~kg} / \mathrm{m}^{3}$ (Figure 2). Alternatively, a last type of model can be derived by considering the central layer as a lower mantle and by fixing the discontinuity at a depth of $560 \mathrm{~km}$. Taking the adiabatic values of 70 and $110 \mathrm{~kg} / \mathrm{m}^{3}$ for $\beta$ in the lower and the upper mantle results in $\alpha=3530$ and $3392 \mathrm{~kg} / \mathrm{m}^{3}$ for the lower and upper mantles, respectively. In Figure 4, we display the four representative models that we have constructed.

\section{Inversion of Data}

[29] For each harmonic degree and order, we can consider two data, the surface topography $h_{\Sigma}(b)$ and the height of the gravity potential $h_{\varphi}(b)$, and a model function, the stress difference $\delta_{l}\left(\sigma_{T}-\sigma_{N}\right)(r)$, which is related to data through relations (29) and (30). This gives the framework to infer the stress difference, which is carried out by a linear inversion, harmonic by harmonic, through a functional least squares approach [e.g., Tarantola and Valette, 1982]. Once the inversion is performed, we can recover the density and the crustal thickness through equations (27) and (28). The a priori covariance of the potential and the topography coefficients is assumed to be diagonal. The regularization of the stress difference is achieved through an a priori covariance kernel of the form $s^{2} \exp \left\{\left(r-r^{\prime}\right)^{2} / 2 L^{2}\right\}$ where $s$ is the standard deviation at radius $r$ and $L$ is the correlation length. We have chosen a null a priori value for the stress difference all over the Moon, in order to obtain the most hydrostatic model compatible with the data.

[30] This inverse problem is strongly underdetermined since a function of the radius is inferred from only two scalar data. However, the model variability is constrained by additional a priori information, which can be imposed through a posteriori controls. The additional information is that the crustal thickness must exceed zero everywhere, the amplitude of the variations in density at the external surface (between 2800 and $3300 \mathrm{~kg} / \mathrm{m}^{3}$ ), the increase of density with depth, and finally, the regularity of the spectra of stress difference and of the spectra of the density. This last point is particulary important. It is well known that spectra of observed fields have a decreasing and regular shape, following a power law, as a function of the harmonic degree $\ell$. Examples of the Earth, Moon, Mars and Venus topography and gravity are given by Bills and Lemoine [1995] or Wieczorek [2007]. Constraining the spectra of the inferred fields to such a regularity indirectly links together the inversions of each harmonic and determines the choice of the parameters of regularization. Among other things, this forces to maintain rather large amplitudes at small $\ell$, yielding large lateral variations in density within the crust. 

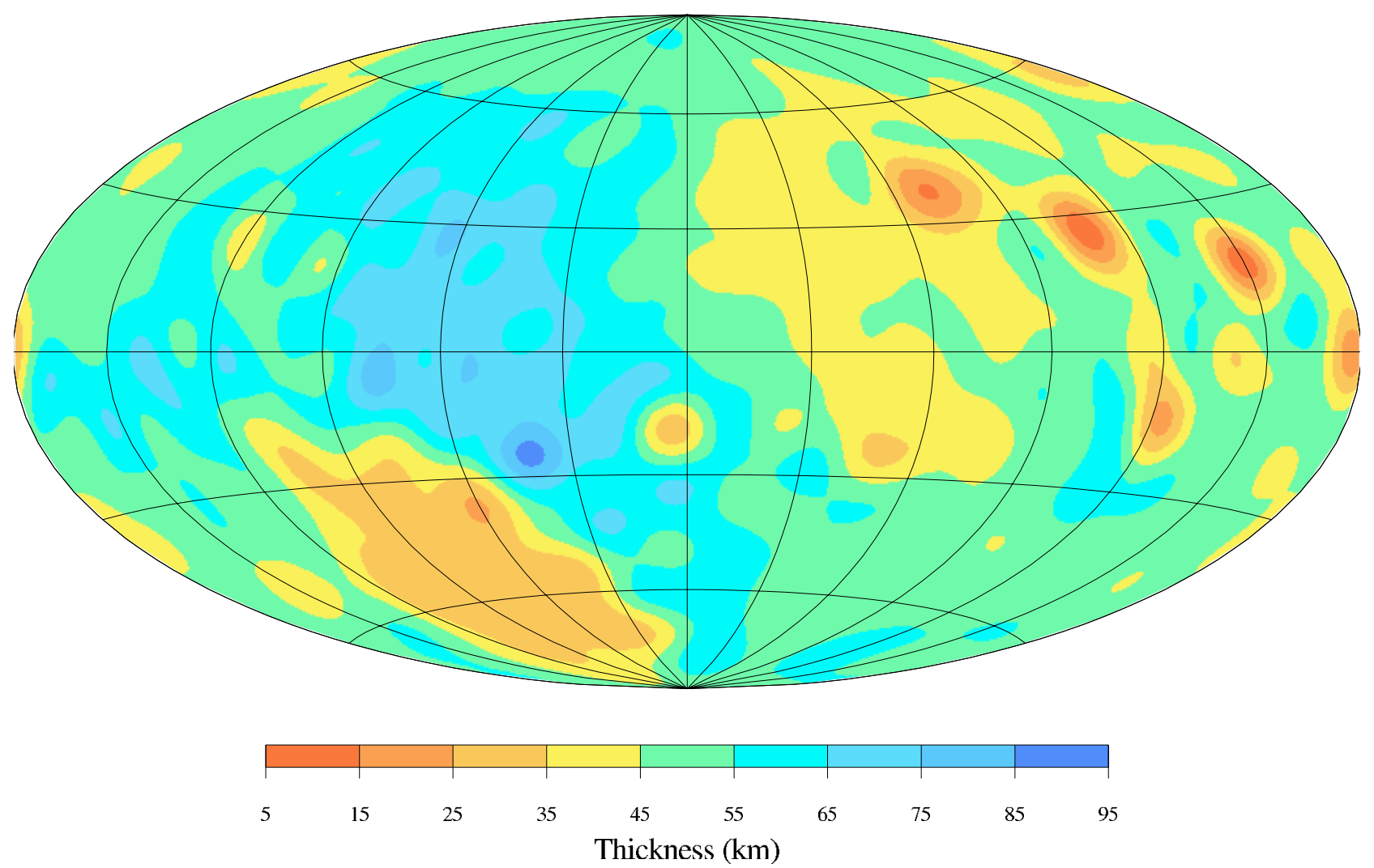

Figure 5. Crustal thickness. The central meridian corresponds to the $90^{\circ} \mathrm{W}$ longitude: the lunar near side at the right and the far side at the left.

[31] By using the different models of mean density that we have described in section 3 , we have checked that the resulting model of lateral variations does not depend upon that choice. This is due to the fact that, regardless of the reference model, the potential kernel $r^{2} \partial_{r} x(r)$ remains very close to $r^{\ell}$. Only the topography of the crust mantle interface, which is proportional to the reference density jump, strongly depends on the reference model. However, this can be easily offset by the choice of the a priori standard deviation in topography.

[32] The topography data that we have used come from the Clementine mission [Smith et al., 1997] and the gravity data from the Lunar Prospector [Konopliv et al., 1998]. Both were first corrected for hydrostatic and permanent tide shape. The inversions have been performed up to degree $\ell=$ 20 , which corresponds to the limit up to which the different gravity models remain well correlated [Konopliv et al., 1998]. One should moreover keep in mind that the farside gravity field is poorly determined because the spacecrafts cannot be observed.

\section{Results}

[33] The results of the inversions are displayed in Figures 5, 6, and 7 through maps of crustal thickness, lateral density variations and stress difference variations in the crust and at the top of the mantle. The corresponding amplitude spectra are drawn in Figures 8 and 9. Because of the $r^{\ell}$ dependence of the kernels, the lateral variations in the deep mantle are not well determined, neither are the depths where they vanish, which mainly depend upon the correlation lengths.

[34] We find large variations in the crustal thickness, from 5 to $90 \mathrm{~km}$ (Figure 5), and we recover the general features obtained by previous authors [e.g., Zuber et al., 1994; Neumann et al., 1996; Wieczorek and Phillips, 1998; Wieczorek, 2007; Hikida and Wieczorek, 2007], namely, the thinning of the crust beneath large impact basins on the nearside, referred as mascons, and the thickening beneath the far side highlands. The large and deep depression of the south pole-Aitken basin is an exception within large basins since it has no mare filling and is associated with no significant free air anomaly. It results in a crustal thickness larger than $25 \mathrm{~km}$ corresponding to a nearly Airy compensated region. By exploring the model space we have found that the crustal thickness is poorly constrained. However, reducing too much the variations in crustal topography would result in unacceptably large density variations at the external surface.

[35] The inferred surface density essentially lies around its mean value $2850 \mathrm{~kg} / \mathrm{m}^{3}$ (Figure 6). The main exceptions correspond to denser materials over the large impact basins, which is in agreement with the filling up of mare with basalt. Because of the a priori correlation lengths that have been used in the inversion process, these lateral variations are not well pronounced, $3140 \mathrm{~kg} / \mathrm{m}^{3}$ instead of the likely value of $3300 \mathrm{~kg} / \mathrm{m}^{3}$, but extend down to the bottom of the crust. In fact, this is not incompatible with the current opinion that the depth of the basaltic mare does not exceed several kilometers [e.g., Wieczorek and Phillips, 1998]. The 

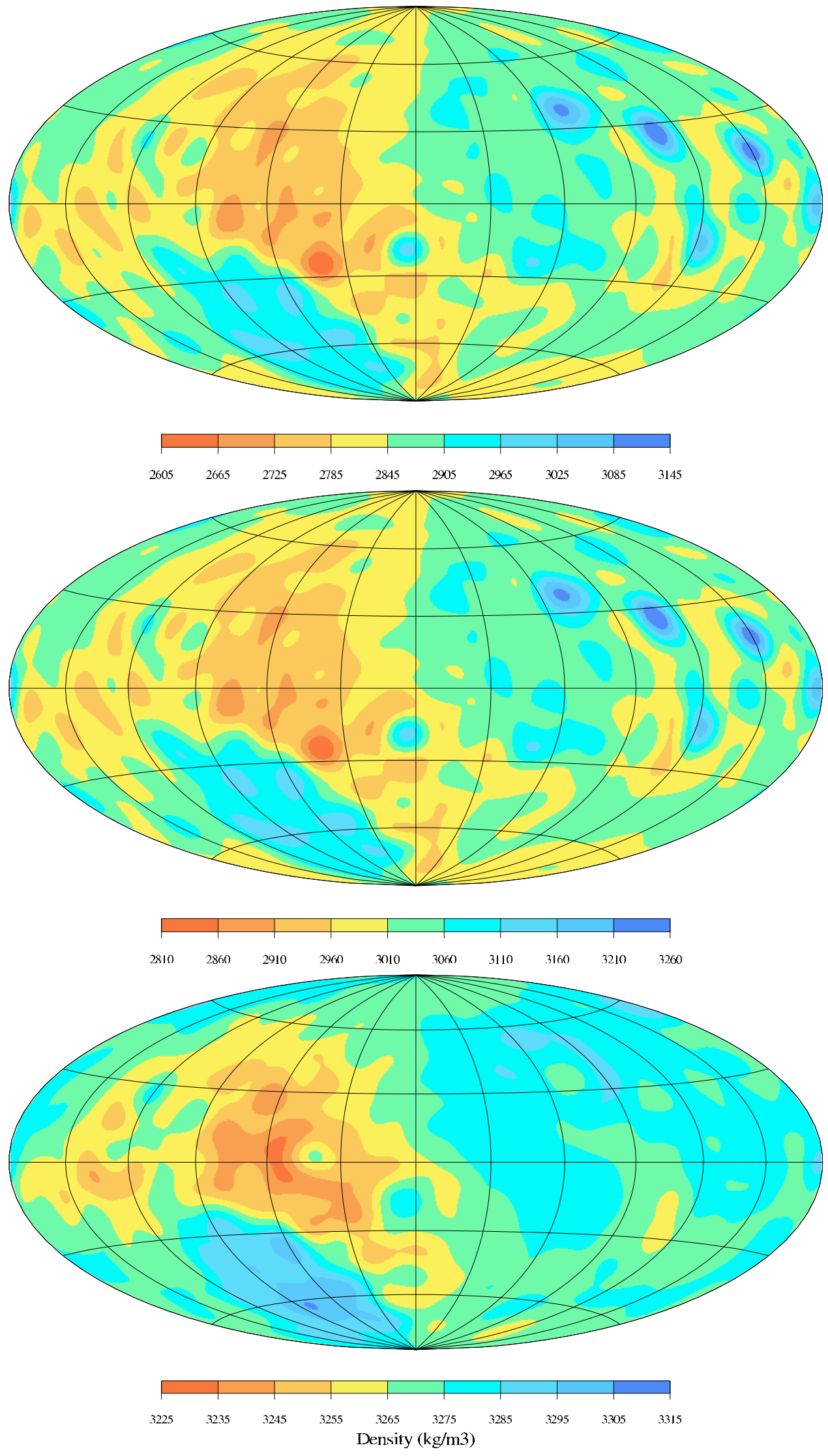

Figure 6. Density $\delta_{\varphi} \rho\left(\mathrm{kg} / \mathrm{m}^{3}\right)$ (top) at the moon surface, (middle) at the bottom of the crust (40 $\mathrm{km}$ depth), and (bottom) in the upper mantle (100 km depth). The projection is the same as for Figure 5. 

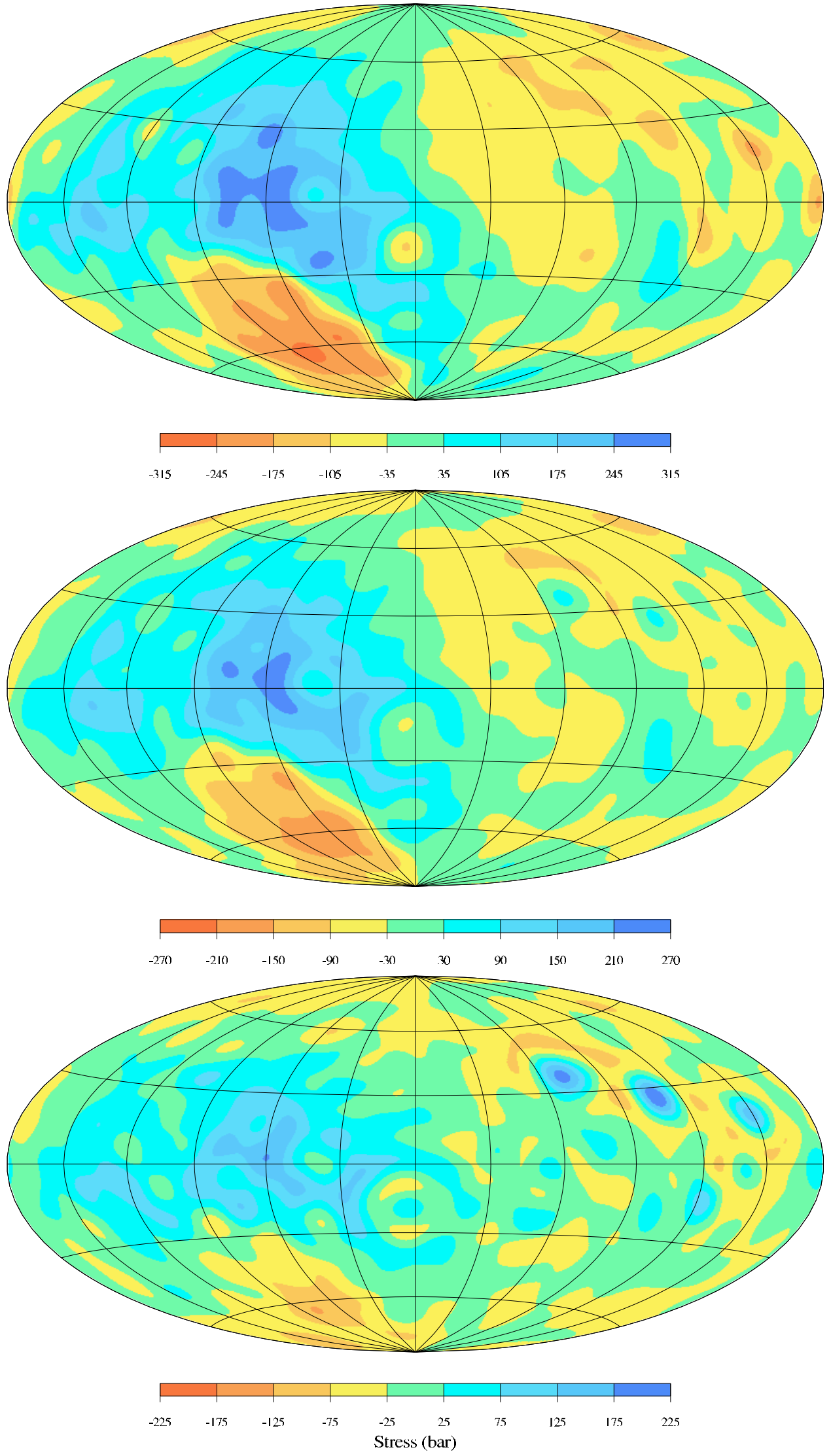

Figure 7. Lateral variations in stress difference $\sigma_{T}-\sigma_{N}$ (top) at the top of the crust, (middle) at the bottom of the crust, and (bottom) in the upper mantle (100 km depth). Positive values indicate vertical compression. The projection is the same as for Figure 5. 
density at the top of the mantle shows smaller variations of $\pm 70 \mathrm{~kg} / \mathrm{m}^{3}$ (Figure 6 , bottom). This amplitude is not well constrained since it is directly related to the radial derivative of the stress difference, the amplitude of which mainly depends on the correlation lengths within the mantle.

[36] At the surface, the state of stress is controlled by the topography according to equation (29). This implies that the topography lows are in a state of lateral compression, while the highlands are in lateral extension. The main feature resides in the far side and corresponds to the great contrast in altitude between the highlands and the south pole-Aitken basin. The variation in stress difference reaches 600 bars (Figure 7) and corresponds to the stress needed to support the $13 \mathrm{~km}$ difference in topography, $\left|\delta_{l}\left(\sigma_{T}-\sigma_{N}\right)\right|=\rho g h_{\Sigma} \simeq$ $2850 \times 1.6 \times 13.10^{3} \mathrm{~Pa} \simeq 590$ bars, an order of magnitude larger than the value proposed by Anderson [1989, p. 39]. At the bottom of the crust the variation in stress shows the same pattern with a smaller amplitude of \pm 250 bars. Note that the maps of stress difference do not account for the mean (degree 0) value, which remains unknown; Figure 7 displays its lateral variations only. At the top of the mantle the signature of the south pole-Aitken basin vanishes. The most prominent feature is the large horizontal tectonic extension beneath the impact basins of the nearside. According to equations (27) and (28), this state of stress can be related to the integrated excess of mass corresponding to the masons above. The magnitude of the stress difference, -130 to 220 bars, is lower than in the crust. The way the stress difference vanishes at depth depends upon the choice of the correlation lengths, and is thus not well determined. Nevertheless, assuming an hydrostatic mantle, while maintaining everywhere a positive thickness of the crust, leads to unacceptably huge values for the density variations within the crust. Therefore tectonic roots do exist within the mantle under the mascons of the nearside. However, since most of the lunar seismicity occur deeper than $700 \mathrm{~km}$ depth, all these stress structure cannot be easily related to moonquakes, which are very sparse in the upper part of the Moon [Nakamura et al., 1982]. Furthermore, we must keep in mind that these results correspond to the particular case of transverse isotropy for the stress tensor.

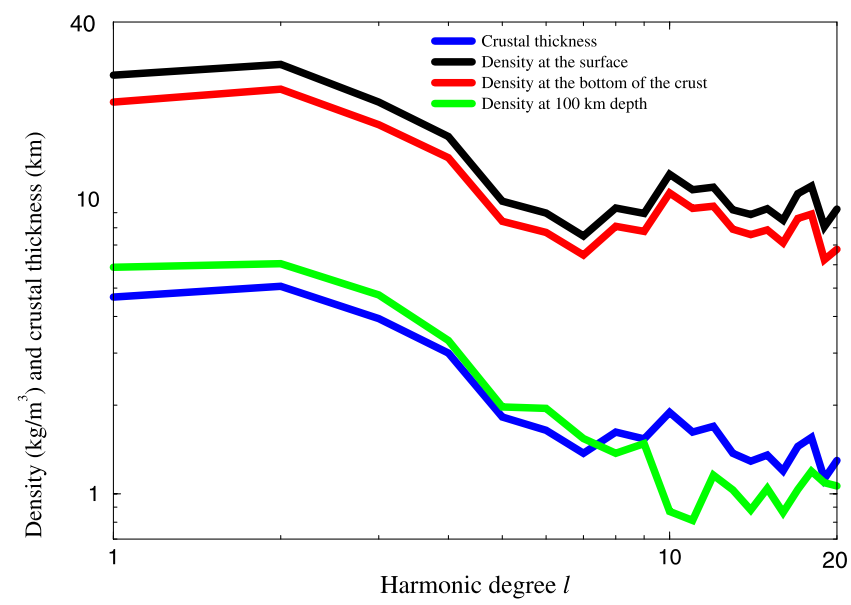

Figure 8. Amplitude spectra of the lateral variations in crustal thickness (blue line) and in density at the top of the crust (black line), at the bottom of the crust (red line), and in the upper mantle (100 km depth, green line).

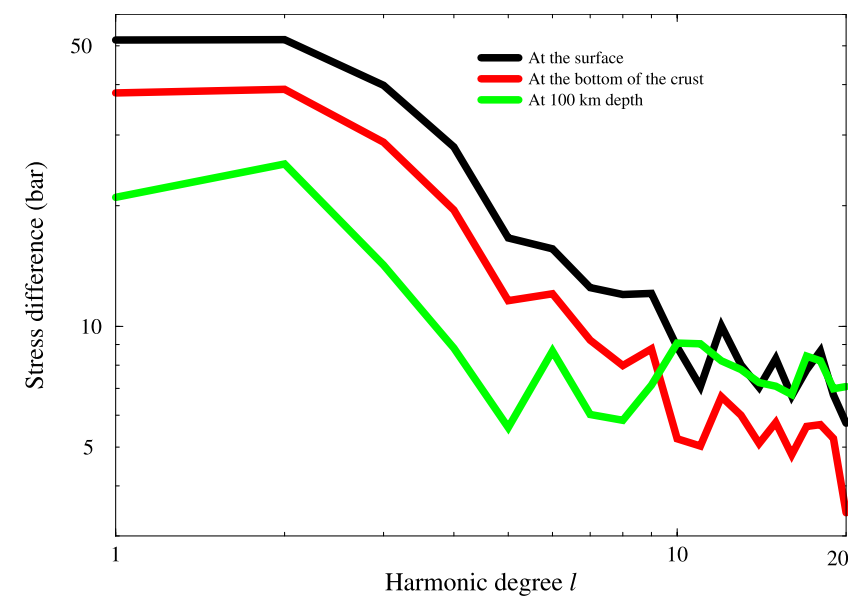

Figure 9. Amplitude spectra of the lateral variations in stress difference $\sigma_{T}-\sigma_{N}$ at the top of the crust (black line), at the bottom of the crust (red line), and in the upper mantle (100 km depth, green line).

In the general framework, the mean of the tangential deviatoric stresses could be smaller due to the introduction of a new degree of freedom. However, the order of magnitude obtained by Dimitrova et al. [2006] and Ghosh et al. [2006] after Flesch et al. [2001] shows that we cannot expect dramatic changes in the mean tangential deviatoric stress.

\section{Conclusion}

[37] We have shown how, by a generalization of Clairaut's equation, one can account for the deviatoric state of stress in gravimetric inversions. Our approach, which can be considered as an attempt to improve isostasy, yields a stress difference compatible with the gravitational potential and the topography. In the case where the stress is assumed to be transversally isotropic, that difference corresponds to the smallest compatible deviatoric stresses. For the Moon, it yields lateral variations in stress difference reaching about 600 bars within the crust and 400 bars in the upper mantle, and permits us to identify tectonic roots in the upper mantle beneath the mascons. Although the currently inferred pattern of crustal thickness seems well established, its amplitude remains poorly constrained. Improvements of the method could be to introduce the nonlinear term coming from topographies in the potential expression, following Chambat and Valette [2005], or to relax the assumption of transverse isotropy.

\section{Appendix A: Proof of Equation (25)}

[38] We perform the Lagrangian perturbation of equilibrium equation (2) corresponding to the Lagrangian displacement field $\xi \mathbf{e}_{r}$, which is radial and follows the stress surfaces, i.e., the surfaces that remain normal to the quasivertical unit vector field $\mathbf{n}$ (see equation (23)).

[39] Let us first remark that the divergence of a tensor given in the form of equation (23) is

$$
\operatorname{divT}=\operatorname{grad} \sigma_{N}+\operatorname{grad}_{T}\left(\sigma_{T}-\sigma_{N}\right)+\left(\sigma_{T}-\sigma_{N}\right) \operatorname{div} \mathbf{P}_{T} .
$$


Second, since gravity is not significantly influenced by reference deviatoric stress, as we could verify for deviatoric stress not exceeding a few kilobars, the reference configuration can be assumed to be hydrostatic: $\sigma_{T}-\sigma_{N}=0$.

[40] The Lagrangian perturbation of $\operatorname{divT}$ can then be expressed as

$$
\begin{aligned}
\delta_{l}(\operatorname{divT})= & \operatorname{grad} \delta_{l} \sigma_{N}-\nabla\left(\xi \mathbf{e}_{r}\right) *\left(\operatorname{grad} \sigma_{N}\right) \\
& +\operatorname{grad}_{T} \delta_{l}\left(\sigma_{T}-\sigma_{N}\right)-2 \delta_{l}\left(\sigma_{T}-\sigma_{N}\right) \mathbf{e}_{r} / r
\end{aligned}
$$

where $\nabla\left(\xi \mathbf{e}_{r}\right) *$ denotes the adjoint, with respect to the usual Euclidean scalar product, of the spatial derivative of $\xi \mathbf{e}_{r}$ and where we have used that in the spherical configuration $\operatorname{div} \mathbf{P}_{T}=-2 \mathbf{e}_{r} / r$.

[41] The perturbation of $\rho \operatorname{grad} \varphi=-\rho \mathbf{g}$ is

$$
\begin{aligned}
\delta_{l}(\rho \operatorname{grad} \varphi) & =-\left(\delta_{l} \rho\right) \mathbf{g}+\rho \operatorname{grad}\left(\delta_{e} \varphi\right)-\rho \nabla \mathbf{g}\left(\xi \mathbf{e}_{r}\right) \\
& =-\left(\delta_{l} \rho\right) \mathbf{g}-\rho \operatorname{grad}(g h)+\rho \nabla\left(\xi \mathbf{e}_{r}\right)^{*}(\mathbf{g}),
\end{aligned}
$$

where we have taken account of the definition of the generalized altitude $h=\xi-h_{\varphi}$, and that $\operatorname{grad}(g \xi)=\nabla\left(\xi \mathbf{e}_{r}\right)^{*}$ $(\mathbf{g})+\nabla \mathbf{g}\left(\xi \mathbf{e}_{r}\right)$.

[42] From relations (A1) and (A2) we deduce that the perturbation of equation (2) is

$$
\begin{aligned}
\operatorname{grad} \delta_{l} \sigma_{N} & +\operatorname{grad}_{T} \delta_{l}\left(\sigma_{T}-\sigma_{N}\right)-2 \delta_{l}\left(\sigma_{T}-\sigma_{N}\right) \mathbf{e}_{r} / r \\
& +\left(\delta_{l} \rho\right) \mathbf{g}+\rho \operatorname{grad}(g h)=0 .
\end{aligned}
$$

Since $\delta_{l} \rho=\delta_{\varphi} \rho+\left(\partial_{r} \rho\right) h$, the sum of the last two terms of equation (A4) can be rewritten as

$$
\left(\delta_{l} \rho\right) \mathbf{g}+\rho \operatorname{grad}(g h)=\left(\delta_{\varphi} \rho\right) \mathbf{g}+\operatorname{grad}(\rho g h),
$$

which gives equation (25).

\section{Appendix B: Proof of Equations (28) and (29)}

[43] From condition (4), we deduce that

$$
\left[\delta_{l}\left(\mathbf{T}\left(\mathbf{n}_{\Sigma}\right) \cdot \mathbf{n}_{\Sigma}\right)\right]=0 .
$$

Let us first consider an interface which is a stress surface, i.e., a surface the normal of which $\mathbf{n}_{\Sigma}$ is an eigendirection of T. The condition (B1) can then be rewritten as

$$
\left[\delta_{l} \sigma_{N}\right]=0
$$

since $\sigma_{N}=\mathbf{T}(\mathbf{n}) \cdot \mathbf{n}$. Taking equation (26) into account, it directly leads to (28):

$$
\left[\delta_{l}\left(\sigma_{T}-\sigma_{N}\right)\right]=-[\rho] g h_{\Sigma}
$$

and, at the outer surface, to (29):

$$
\delta_{l} \sigma_{N}(b)=0 \text { and } \delta_{l}\left(\sigma_{T}-\sigma_{N}\right)(b)=-\rho g h_{\Sigma}(b) \text {. }
$$

Let us now turn to the general case for which the interface is not assumed to be a stress surface. To relate condition
(B1) to $\delta_{l} \sigma_{N}$, we use relation (7) and write, correct to first order, that

$$
\begin{aligned}
{\left[\delta_{l}\left(\mathbf{T}\left(\mathbf{n}_{\Sigma}\right) \cdot \mathbf{n}_{\Sigma}\right)\right] } & =\left[\delta_{l} \sigma_{N}+\operatorname{grad} \sigma_{N} \cdot \mathbf{e}_{r}\left(\xi_{\Sigma}-\xi\right)\right] \\
& =\left[\delta_{l} \sigma_{N}-\rho g\left(h_{\Sigma}-h\right)\right],
\end{aligned}
$$

where $h_{\Sigma}$ is the altitude of the interface above the equipotential surface while $h$ is the altitude of the corresponding stress surface above the same equipotential surface. Upon making use of equation (26), it leads, once again, to equation (B3), that is equation (28).

[44] Acknowledgments. We thank the Editor and the reviewers for helpful comments and suggestions. Jan Matas helped us to determine the value of iron density at the lunar center conditions. This work has been partially supported by grants from INSU-CNRS, France.

\section{References}

Anderson, D. L. (1989), Theory of the Earth, Blackwell Sci., Oxford, U. K. Backus, G. E. (1967), Converting vector and tensor equations to scalar equations in spherical coordinates, Geophys. J. R. Astron. Soc., 13, $71-101$.

Bills, B. G., and F. G. Lemoine (1995), Gravitational and topographic isotropy of the Earth, Moon, Mars and Venus, J. Geophys. Res., 100, $26,275-26,295$

Bills, B. G., and D. P. Rubincam (1995), Constraints on density models from radial moments: Applications to Earth, Moon and Mars, J. Geophys. Res., 100, 26,305-26,315.

Boehler, R., N. von Bargen, and A. Chopelas (1990), Melting, thermal expansion, and phase transitions of iron at high pressures, J. Geophys. Res., 95, 21,731-21,736.

Chambat, F. (1996), Figure de la Terre: Gravimétrie, régime de contraintes et vibrations propres, thèse de Doctorat $(\mathrm{PhD})$, Paris 7 Univ., Paris.

Chambat, F., and B. Valette (2001), Mean radius, mass and inertia for reference Earth's models, Phys. Earth. Planet. Inter., 124/3-4, 237-253.

Chambat, F., and B. Valette (2005), Earth gravity up to second order in topography and density, Phys. Earth. Planet. Inter., 151, 10-89.

Dahlen, F. A. (1981), Isostasy and the ambient state of stress in the oceanic lithosphere, J. Geophys. Res., 86, 7801-7807.

Dahlen, F. A. (1982), Isostatic geoid anomalies on a sphere, J. Geophys. Res., 87, 3943-3947.

Dimitrova, L. L., W. E. Holt, A. J. Haines, and R. A. Schultz (2006), Toward understanding the history and mechanisms of Martian faulting: The contribution of gravitational potential energy, Geophys. Res. Lett., 33, L08202, doi:10.1029/2005GL025307.

Flesch, L. M., A. J. Haines, and W. E. Holt (2001), The dynamics of the India-Eurasia collision zone, J. Geophys. Res., 106, 16,435-16,460.

Ghosh, A., W. E. Holt, A. J. Haines, and L. M. Flesch (2006), Gravitational potential energy of the Tibetan Plateau and the forces driving the Indian Plate, Geology, 34, 321-324

Hikida, H., and M. A. Wieczorek (2007), Crustal thickness of the Moon: New constraints from gravity inversions using polyhedral shape models, Icarus, 192(1), 150-166, doi:10.1016/j.icarus.2007.06.015.

Kaula, W. (1968), Introduction to Planetary Physics: The Terrestrial Planets, John Wiley, New York.

Khan, A., and K. Mosegaard (2002), An inquiry into the lunar interior: A nonlinear inversion of the Apollo lunar seismic data, J. Geophys. Res., 107(E6), 5036, doi:10.1029/2001JE001658.

Khan, A., K. Mosegaard, and K. L. Rasmussen (2000), A new seismic velocity model for the Moon from a Monte Carlo inversion of the Apollo seismic data, Geophys. Res. Lett., 27, 1591-1594.

Kiefer, W. S. (1997), Gravity models for lunar mascon basins: Distribution and thickness of mare basalts and basin ejecta, paper presented at 28th Annual Lunar and Planetary Science Conference, Lunar Planet. Inst., Houston, Tex.

Konopliv, A. S., A. B. Binder, L. L. Hood, A. B. Kucinskas, W. L. Sjogren, and J. G. Williams (1998), Improved gravity field of the Moon from Lunar Prospector, Science, 281, 1476-1480.

Lemoine, F. G., D. E. Smith, M. T. Zuber, G. A. Neumann, and D. D. Rowlands (1997), A 70th degree lunar gravity model (GLGM-2) from Clementine and other tracking data, J. Geophys. Res., 102, 16,33916,359 .

Lognonné, P., J. Gagnepain-Beyneix, and H. Chenet (2003), A new seismic model of the Moon: Implications for structure and thermal evolution and formation of the Moon, Earth Planet. Sci. Let., 211, 27-44. 
Mohr, P. J., and B. N. Taylor (2005), CODATA Recommended Values of the fundamental physical constants: 2002, Rev. Mod. Phys., 77(1).

Nakamura, Y. (1983), Seismic velocity structure of the lunar mantle, J. Geophys. Res., 88, 677-686.

Nakamura, Y., G. V. Latham, and H. J. Dorman (1982), Apollo lunar seismic experiment - Final summary, Proc. Lunar Planet, Sci. Conf. 13th, Part 1, J. Geophys. Res., 87, suppl., A117-A123.

Neumann, G. A., M. T. Zuber, D. E. Smith, and F. G. Lemoine (1996), The Lunar crust: Global structure and signature of major basins, J. Geophys. Res., 101, 16,841-16,843.

Piersanti, A., L. Boschi, and A. M. Dziewonski (2001), Estimating lateral structure in the Earth's outer core, Geophys. Res. Lett., 28, 1659-1662.

Smith, D. E., M. T. Zuber, G. A. Neumann, and F. G. Lemoine (1997), Topography of the Moon from the Clementine Lidar, J. Geophys. Res., $102,1591-1611$.

Solomon, S. C., and M. N. Toksöz (1973), Internal constitution and evolution of the Moon, Phys. Earth. Planet. Inter., 7, 15-38.

Stieltjes, T. J. (1884), Quelques remarques sur la variation de la densite dans l'intérieur de la Terre, Arch. Néerl. Sci. Soc. Holl., 19, 435-460.

Tarantola, A., and B. Valette (1982), Generalized nonlinear inverse problems solved using the least squares criterion, Rev. Geophys., 20, 219232.

Valette, B. (1987), Spectre des oscillations libres de la Terre; Aspects mathématiques et géophysiques, thèse de Doctorat d'Etat, Univ. Pierre et Marie Curie Paris 6, Paris

Valette, B. (2000), Bounds of the density moments inside the Earth or a planet, C. R. Acad. Sci., 330, 235-240.
Valette, B., and F. Chambat (2004), Relating gravity, density, topography and state of stress inside a planet, in V Hotine-Marussi Symposium on Mathematical Geodesy, IAG Symp. Ser., vol. 127, pp. 301-308, edited by F. Sanso, pp. 301-3-8, Springer, Berlin.

Wieczorek, M. A. (2007), The gravity and topography of the terrestrial planets, in Treatise on Geophysics, vol. 10, Planets and Moons, edited by T. Spohn, pp. 165-206, Elsevier, New York, doi:10.1016/B978044452748-6/00156-5.

Wieczorek, M. A., and R. J. Phillips (1997), The structure and compensation of the lunar highland crust, J. Geophys. Res., 102, 10,933-10,943.

Wieczorek, M. A., and R. J. Phillips (1998), Potential anomalies on a sphere: Applications to the thickness of the lunar crust, J. Geophys. Res., 103, 1715-1724.

Williams, J. G., D. H. Boggs, C. F. Yoder, J. T. Ratcliff, and J. O. Dicke (2001), Lunar rotational dissipation in solid body and molten core, J. Geophys. Res., 106, 27,933-27,968.

Zuber, M., D. Smith, F. Lemoine, and G. Neumann (1994), The shape and internal structure of the Moon from the Clementine mission, Science, $266,1839-1843$

F. Chambat, LST, ENS Lyon, 46 Allée d'Italie, F-69364 Lyon Cedex 07, France. (frederic.chambat@ens-lyon.fr)

B. Valette, LGIT, IRD, Université de Savoie, F-73376 Le Bourget-du-Lac Cedex, France. (bernard.valette@univ-savoie.fr) 